\title{
Patients' preferences in treatment for neovascular age-related macular degeneration in clinical routine
}

\author{
Robert Finger, ${ }^{1,2}$ Andrea E Hoffmann, ${ }^{3}$ Eva K Fenwick, ${ }^{1}$ Armin Wolf, ${ }^{3}$ \\ Anselm Kampik, ${ }^{3}$ Marcus Kernt, ${ }^{3}$ Aljoscha S Neubauer, ${ }^{3}$ Christoph Hirneiss ${ }^{3}$
}

${ }^{1}$ Center for Eye Research Australia, Royal Victorian Eye and Ear Hospital, University of Melbourne, Melbourne, Australia

${ }^{2}$ Department of Ophthalmology, University of Bonn, Bonn,

Germany

${ }^{3}$ Department of Ophthalmology, Ludwig-Maximilians University, Munich, Germany

\section{Correspondence to}

Dr Christoph Hirneiss

Augenklinik der

Ludwig-Maximilians-Universität,

Mathildenstr. 8, D-80336

München, Germany;

christoph.hirneiss@med.

uni-muenchen.de

Parts of this study were presented at the annual meeting of the Association for Research in Vision and Ophthalmology, May 2010 in Fort Lauderdale, Florida, USA

Accepted 6 April 2012 Published Online First 25 April 2012

\section{ABSTRACT}

Purpose To assess the effect of ranibizumab treatment for neovascular age-related macular degeneration (nvAMD) on patients' preferences and vision-related quality of life (VROoL) in a routine clinical setting. Methods 55 treatment naïve patients were examined before and after the initial upload of three monthly injections of $0.5 \mathrm{mg}$ ranibizumab. VROoL was assessed using a Rasch-adjusted NEI-VFQ-25. Time trade-off (TTO), standard gamble, a visual analogue scale and the European Quality of Life Questionnaire (EO-5D) were used to calculate utilities, and multiple logistic regression models were conducted to determine independent factors associated with utilities.

Results Mean \pm SD age was $75 \pm 7$ years, and 40 patients $(73 \%)$ were female. Mean \pm SD best-corrected visual acuity of the treated eye increased from 20/80 at baseline (logMAR $0.60 \pm 0.35$ ) to 20/63 (logMAR $0.52 \pm 0.36 ; p=0.020$ ) at follow-up after three injections. Utility score increases ranged from 2 utils (standard gamble anchored for death) up to 6.6 utils (EQ-5D German $T T 0, p=0.023$ ) and visual functioning improved (Rasch adjusted composite NEI-VFO score $50 \pm 21$ to $54 \pm 21, p=0.042$ ). Whether the worse or better eye was treated was not significantly associated with improvements in utility or VROoL, whereas VA improvement in the treated eye was associated with an increase in utility (TTO, $p=0.020$ ).

Conclusions TTO performed best in this sample of elderly nvAMD patients undergoing anti-VEGF therapy. Better or worse eye treatment was not associated with a change in reported utilities or visual functioning in patients with newly diagnosed nvAMD. Directly elicited, vision-specific utilities gained with TTO seem to be sensitive to a change in vision status.

\section{INTRODUCTION}

A positive effect of ranibizumab treatment on patients' vision related quality of life (VROoL) in neovascular (nv) age-related macular degeneration (AMD) has been reported in several studies. ${ }^{12}$ One way to assess the impact of treatment on patients' QoL and, in particular, the satisfaction derived from a specific health outcome, is to assess utilities. Utility in a general sense refers to the preference an individual or society may have for any particular set of health outcomes. Utilities are a crucial measure for cost-benefit analyses and are used widely by policy planners for allocation of healthcare resources. ${ }^{3}$ Commonly used tools to determine utilities in ophthalmology include the time tradeoff method (TTO), standard gamble (SG) and the
European Quality of Life Questionnaire (EQ-5D). ${ }^{4}$ The different approaches have different limitations. TTO and SG are affected by time and duration effects, and SG by attitudes to risk as well as anchoring issues, while generic health-related $\mathrm{OoL}$ utilities, especially the EQ-5D, may not be sensitive to the specific effects of vision or vision impairment since they often do not contain vision-related content. Utility values and remaining life years are then used to calculate quality adjusted life years (OALYs), which are the basis of economic evaluations in healthcare.

When considering healthcare resource allocation for treatment of nvAMD, economic evaluations frequently differentiate between treatment of the first (worse) and second (better) eye, assuming a differential impact on patients' preferences (utilities) and QoL, and thus differing cost implications and cost-benefit ratios. ${ }^{5}$ However, no data on patient reported preferences for anti-VEGF treatment of nvAMD assessed by standard utility instruments are currently available. Therefore, we investigated the effect of ranibizumab treatment for nvAMD on patients' preferences and VRQoL in a routine clinical setting using a range of utility methods, assessing their performance and appropriateness.

\section{PATIENTS AND METHODS}

Patients were recruited from the outpatient clinic at the Department of Ophthalmology, University of Munich, between September 2009 and March 2010. Approval was obtained from the Institutional Review Board of the University of Munich. All patients gave signed informed consent for study participation before enrolment and start of treatment. The study adhered to the tenets of the Declaration of Helsinki.

\section{Patients and intervention}

Only treatment naïve, newly diagnosed patients with nvAMD were included. The diagnosis of nvAMD was confirmed by fluorescein angiography. Patients received three monthly intravitreal injections of $0.5 \mathrm{mg}$ ranibizumab. Participants underwent a full ophthalmological examination pre- and post-treatment, including best-corrected visual acuity (BCVA) using retro-illuminated $\log M A R$ ETDRS charts (Lighthouse International, New York, USA), fundus photography and optical coherence tomography. VRQoL and patients' preferences were recorded at baseline and follow-up after the third ranibizumab injection, in order to ensure that all patients underwent the same 
treatment regime and that the treatment effect was likely to be at its maximum level possible.

\section{Vision-related quality of life}

The National Eye Institute Visual Function Questionnaire 25 Item (NEI-VFO-25), a well evaluated tool available in German, ${ }^{6}$ is one of the most widely used QoL scales in ophthalmology. In the current study, we performed Rasch analysis to assess the measurement properties of the German NEI-VFO-25.

\section{Psychometric validation of the German NEI-VFO-25}

In brief, Rasch analysis is a modern psychometric method that mathematically describes the interaction between respondents and test items. The Rasch model states that the probability of a correct response is a logistic function of the difference between person ability (person measure) and item difficulty (item measure), and applies a strict model which the pattern of participants' responses should satisfy. Raw ordinal scores are thus transformed into data that approximate interval-level measurement (expressed in log of the odds units, or logits). The specific methodology employed has been described in detail elsewhere. ${ }^{7}$ We performed Rasch analysis using Winsteps software (V.3.68; Chicago, Illinois, USA).

\section{Patient-reported preferences}

Health state utilities depend on the individuals' relative preferences for different health states. The instruments used to assess the patients' preferences in this study included TTO, SG and the Euro-QoL (EQ-5D). ${ }^{4}$

\section{Time trade-off}

Within the TTO utility assessment, the respondents are asked to trade-off life for gains in health status. In ophthalmology the perfect health state is set at full visual function. The utility value is calculated as a ratio of the highest state of QoL equalling a utility value of 1.0 , whereas the worst utility value is $0 .^{8}$

TTO was conducted as follows. The first question to respondents was "How many years do you still expect to live?", followed by the second question "Assuming that there was a technology which restores you to full vision, what is the maximum number of remaining years of life you would be willing to give up if you could receive this technology and have normal vision in both eyes for the rest of your life?" Utilities were calculated as: (expected life years - years willing to trade off)/expected life years.

\section{Standard gamble}

A typical SG lottery asks a person to choose between experiencing a specific health state with certainty for the rest of their life and a lottery, usually described as a medical intervention, with two possible outcomes: an immediate return to perfect health with probability $\mathrm{p}$ or immediate death with probability $(1-p)$. The probabilities of the two outcomes are repeatedly adjusted until the person indicates equal preferences between the certain health state and the lottery. The utility weight for the certain health state is equal to the individually adjusted probability $\mathrm{p}$.

In our study, we performed two SG approaches which were anchored for two worst lottery outcome health states: death and blindness. Two anchor points were chosen as the readiness to take risks depends on the worst outcome. ${ }^{9}$ The SG utilities were estimated using a computer-based preference assessment interview platform (X-trade).

\section{E0-5D and visual analogue scale}

The EQ-5D is a generic multi-attribute utility instrument developed by the EuroOoL group, ${ }^{10}$ comprising five dimensions-mobility, self-care, usual activities, pain/discomfort and anxiety/depression - and three severity levels. The EQ-5D also includes a visual analogue scale (VAS), which comprises a line with numbers between 0 (worst health-state) and 100 (best health-state). The raw data of the EQ-5D values were converted into utilities using the suggested conversion algorithms anchored for the VAS for a European (European VAS) and German (German VAS) collective and by TTO for a German collective (German TTO).

\section{Statistical analyses}

SPSS V.19.0 was used to analyse the data. Descriptive statistical analyses were performed to characterise the participants' sociodemographic, clinical, utility and VRQoL data, stratified by better or worse eye treated. Correlational analyses (bivariate and intraclass) were used to explore employed utility measures. Instruments were grouped into directly elicited, vision-specific utilities (TTO and SG, including different anchors), general utilities (different weights for the EQ-5D) and VRQoL estimates (VAS and Rasch scored NEI-VFO-25). Fully-adjusted logistic regression models were conducted to determine the independent factors associated with patient-reported preferences (utilities) and VRQoL. Visual acuity was categorised into two categories: normal presenting vision in the better eye $(<0.4 \log \mathrm{MAR})$; and visual impairment and blindness (logMAR $\geq 0.4)$. All tests were considered to be statistically significant at an adjusted level of $\mathrm{p}<0.05$

\section{RESULTS}

\section{Clinical characteristics}

A total of 55 patients with newly diagnosed and treatment naïve nvAMD participated in the study (table 1 ). Mean \pm SD age was $75 \pm 7$ years, and 40 patients $(73 \%)$ were female. The mean BCVA of the treated eye increased from 20/80 at baseline $(\log M A R \quad 0.60 \pm 0.35)$ to $20 / 63(\log M A R \quad 0.52 \pm 0.36 ; p=0.020)$ at follow-up after three injections (table 1). Mean BCVA of the

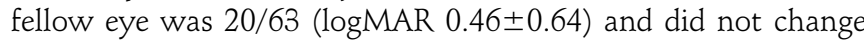
during the study period. VA significantly improved only in treated worse eyes (logMAR $0.69 \pm 0.35$ to $0.57 \pm 0.38$; $p=0.013$ ). Visual acuity in treated better seeing eyes remained stable (logMAR 0.43 \pm 0.31 ).

Utility scores increased for the whole sample pre- and posttreatment from 2 utils (SG anchored for death) up to 6.6 utils (EQ-5D German TTO). The only statistically significant utility value increase for the whole sample was found with the EQ-5D German TTO utilities and the VAS measurement ( $p=0.023$ and $p=0.001$, respectively, table 1 ). There was no measurable change in utility scores over time within or between the worst or better eye treatment groups except for the VAS measurement which improved in patients whose worse eye was treated $(p \leq 0.01$; table 1).

\section{Psychometric evaluation of the NEI-VFO}

After a number of revisions of the original scale, including collapsing response categories to three and splitting the multidimensional overall scale into two scales, a visual functioning and socioemotional well-being subscale, the visual functioning subscale of the NEI-VFQ-25, demonstrated good measurement characteristics as shown in table 2. Discriminant ability of the socioemotional scale could not be improved by further deletion 
Table 1 Characteristics of the sample $(n=55)$

\begin{tabular}{|c|c|c|c|c|c|c|c|c|}
\hline & & \multicolumn{2}{|l|}{$\begin{array}{l}\text { Total sample } \\
\mathrm{n}=\mathbf{5 5}\end{array}$} & $\begin{array}{l}\text { Worse eye treated } \\
n=36\end{array}$ & \multicolumn{2}{|c|}{$\begin{array}{l}\text { Better eye treated } \\
n=18\end{array}$} & & p Value $\S$ \\
\hline \multicolumn{9}{|l|}{ Proportion } \\
\hline \multicolumn{9}{|l|}{ Gender } \\
\hline Female & & $40(72.7 \%)$ & \multicolumn{2}{|c|}{$24(66.7 \%)$} & \multicolumn{2}{|c|}{$15(83.3 \%)$} & & \\
\hline \multicolumn{2}{|c|}{ Age (years), mean $\pm S D$} & $75.5 \pm 6.9$ & \multicolumn{2}{|c|}{$75.6 \pm 7.0$} & \multicolumn{2}{|c|}{$75.2 \pm 7.0$} & \multicolumn{2}{|r|}{$0.837 \ddagger$} \\
\hline & BL & FU & $\mathrm{BL}$ & FU & $\overline{B L}$ & FU & $B$ & FU $\neq$ \\
\hline \multicolumn{9}{|c|}{ Treated eye VA (logMAR) } \\
\hline Mean $\pm S D$ & $0.60 \pm 0.35$ & $0.52 \pm 0.36$ & $0.69 \pm 0.35$ & $0.57 \pm 0.38$ & $0.43 \pm 0.31$ & $0.43 \pm 0.32$ & & \\
\hline p Value ${ }^{*}$ & 0.020 & & 0.013 & & 0.987 & & 0.011 & 0.194 \\
\hline \multicolumn{9}{|c|}{ Other eye VA (logMAR) } \\
\hline p Value ${ }^{*}$ & 0.469 & & 0.239 & & 0.604 & & 0.004 & 0.006 \\
\hline \multicolumn{9}{|c|}{ Standard gamble death } \\
\hline Mean $\pm S D$ & $0.95 \pm 0.12$ & $0.97 \pm 0.08$ & $0.95 \pm 0.13$ & $0.98 \pm 0.05$ & $0.94 \pm 0.10$ & $0.96 \pm 0.12$ & & \\
\hline p Value ${ }^{*}$ & 0.364 & & 0.352 & & 0.782 & & 0.858 & 0.511 \\
\hline Standard gam & dness & & & & & & & \\
\hline Mean $\pm S D$ & $0.95 \pm 0.12$ & $0.98 \pm 0.06$ & $0.95 \pm 0.12$ & $0.98 \pm 0.05$ & $0.95 \pm 0.10$ & $0.98 \pm 0.08$ & & \\
\hline p Value* & & 0.135 & & 0.231 & & 0.384 & 0.981 & 0.979 \\
\hline тто & & & & & & & & \\
\hline Mean $\pm S D$ & $0.89 \pm 0.16$ & $0.92 \pm 0.16$ & $0.87 \pm 0.16$ & $0.90 \pm 0.17$ & $0.95 \pm 0.03$ & $0.95 \pm 0.04$ & & \\
\hline p Value ${ }^{*}$ & 0.326 & & 0.368 & & 0.904 & & 0.074 & 0.373 \\
\hline EQ-5D German & & & & & & & & \\
\hline Mean $\pm S D$ & $0.79 \pm 0.26$ & $0.86 \pm 0.20$ & $0.80 \pm 0.27$ & $0.87 \pm 0.20$ & $0.78 \pm 0.25$ & $0.83 \pm 0.22$ & & \\
\hline
\end{tabular}

Emboldened values indicate statistical significance.

*Two-tailed paired t-test.

†Pearson's $\chi^{2}$

‡Independent samples two-tailed t-test.

$\S p$ Value refers to the difference in baseline and follow-up scores between the worse and better eye treatment groups.

EQ-5D, European Quality of Life Instrument, 5 dimensions; NEI-VFO, National Eye Institute Visual Functioning Questionnaire; TTO, time trade-off; VA, visual acuity; VAS, visual analogue scale.

or inclusion of items. Thus the socioemotional scale was only used in the correlational analyses (table 3 ) but not in any of the regression models. The process is outlined in a previous Rasch analysis of the NEI-VFO-25. ${ }^{11}$ To facilitate the interpretation of the person measure scores, they were recalibrated from a negative-positive logit scale to range between 0 and 100 following
Rasch analysis. Visual functioning improved from baseline to follow-up in the whole sample by 4.0 points on the transformed VFO scale $(50 \pm 21$ to $54 \pm 21, p=0.042$; table 1$)$. As with the utility measures, no differences were found for visual functioning over time within or between the better and worse eye treatment groups.

Table 2 The fit parameters of the German NEI-VFO 25 Item compared to the Rasch model

\begin{tabular}{|c|c|c|c|c|}
\hline \multirow[b]{2}{*}{ Parameters } & \multirow[b]{2}{*}{ Rasch model } & \multirow{2}{*}{$\begin{array}{l}\text { NEI-VF0-25 } \\
\text { overall score }\end{array}$} & \multicolumn{2}{|c|}{ NEI-VF0-25 Rasch guided subscales } \\
\hline & & & Visual functioning & Socioemotional \\
\hline Item no. & & $1-25$ & $5-14$ & $3,17,18,20-24$ \\
\hline Number of misfitting items & 0 & 9 & 0 & 0 \\
\hline Person separation & $>2.0$ & 2.16 & 2.21 & 1.58 \\
\hline Person reliability & $>0.8$ & 0.82 & 0.83 & 0.71 \\
\hline Person mean & 0 & 1.13 & 1.47 & 2.69 \\
\hline $\begin{array}{l}\text { Principal components analysis } \\
\text { (eigenvalue for first contrast) }\end{array}$ & $<2.0$ & 3.8 & 1.6 & 1.8 \\
\hline Variance by the first factor & $>50 \%$ & $49.6 \%$ & $53.5 \%$ & $68.1 \%$ \\
\hline
\end{tabular}


Table 3 Correlations of utility measures and visual acuity at baseline (bivariate Pearson's correlation coefficients)

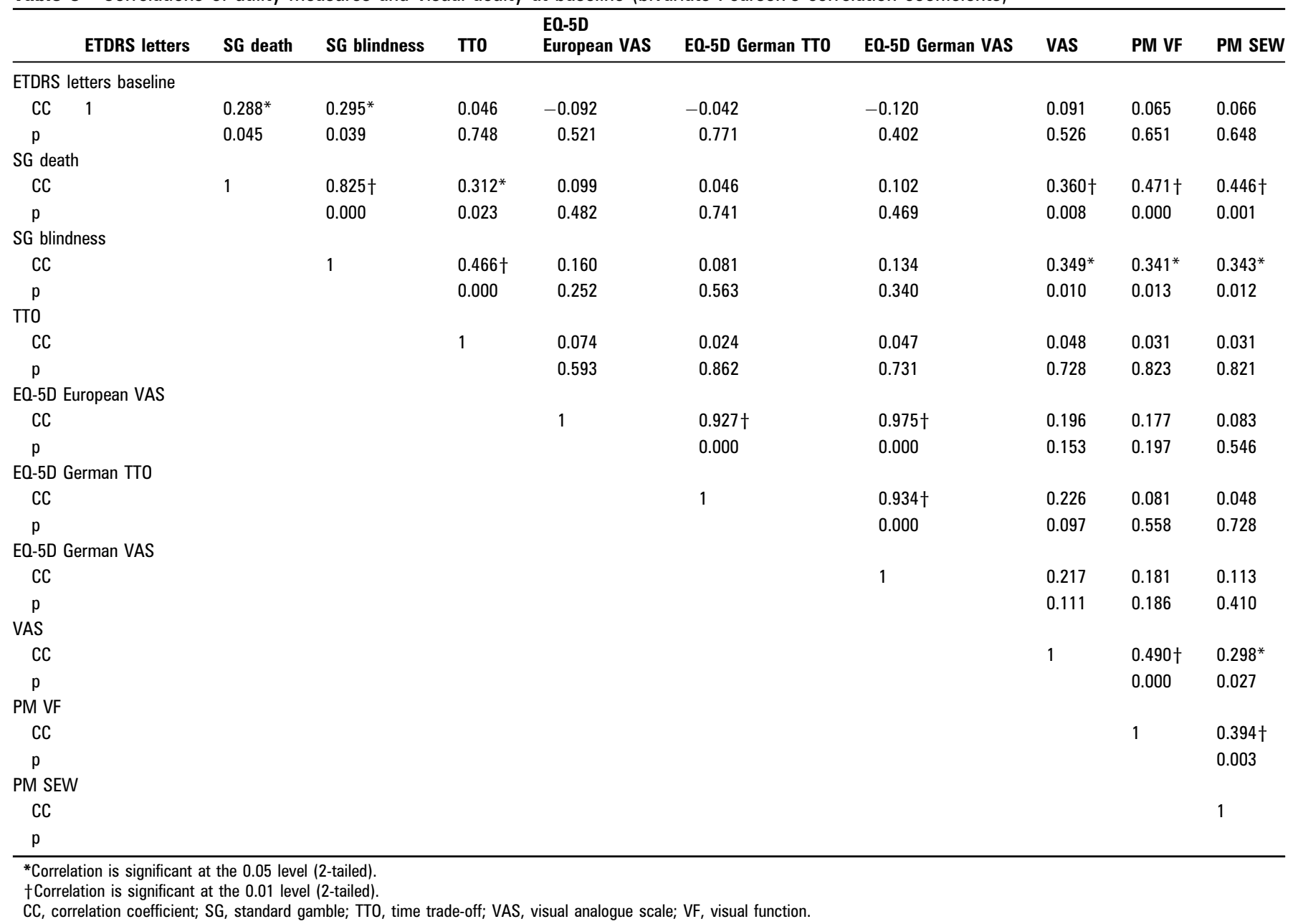

\section{The relation of reported utilities}

All vision-specific utilities, namely the SG anchored for death and blindness and the TTO, were highly correlated using Pearson's correlation coefficient (table 3 ) and intraclass correlation $(\mathrm{r}>0.5 ; \mathrm{p}<0.001$ for all). All three measures showed similar ceiling effects, but only SG measures were correlated to VA, VAS and person measures (visual functioning and socioemotional well-being, table 3). As the SG anchor for blindness cannot be used to calculate OALYs, we only used the SG anchored for death for subsequent analyses.

The three different weightings for the EQ-5D data were highly correlated with each other; however, they correlated to no other clinical measures. Ceiling effects were present but less marked than for SG and TTO. In subsequent analyses, only the German TTO weighting was used.

The Rasch guided person measures for the visual functioning and socioemotional well-being subscales of the NEI-VFQ-25 and the EQ-5D based VAS rating were all inter-correlated and correlated to both SG ratings, but were not correlated with other clinical measures (table 3 ).

\section{The impact of treatment on patients' preferences}

We created binary variables for all utility scores at follow-up, categorising the change into improvement (including stable scores, difference to baseline $\geq 0$ ) and worsening (difference to baseline $<0$ ). Logistic regression models were used to examine the association between an improvement in patient reported prefer- ences and ranibizumab treatment for nvAMD. An improvement of vision in the treated eye was significantly associated with an improvement in the TTO score $(p=0.020$; table 4$)$. However, whether the better or worse eye was treated was not significantly associated with any of the utility measures. Age was significantly associated with reported differences in SG anchored for death $(\mathrm{p}=0.010)$ and the EQ-5D German TTO ( $p=0.038$; table 4). An improvement in visual functioning as measured with the NEIVFO-25 subscale was significantly associated with an improvement in reported scores for SG anchored for death $(p=0.025)$.

\section{DISCUSSION}

In this sample, ranibizumab treatment for nvAMD led to an improvement in visual acuity of the treated eye, patient reported visual functioning and utilities as measured with TTO and the EQ-5D anchored for German TTO. In multivariate analyses, better or worse eye treatment was not significantly associated with any utility measures, whereas an improvement of vision in the treated eye was significantly associated with increased TTO utilities. SG utilities (both anchors) were highly concordant but were not responsive to treatment in this sample. This has important implications for economic evaluations of ophthalmic interventions as it suggests that vision-specific, directly elicited utilities collected with TTO may be most sensitive to a change in vision status. Generally, responsiveness of utility instruments to change over time, that is after an intervention, remains a challenge to be addressed. 
Table 4 Binary logistic regression models for change ('better or stable' vs 'worse') in reported utility scores

\begin{tabular}{|c|c|c|c|c|c|c|c|c|}
\hline & \multicolumn{2}{|l|}{ Standard gamble death } & \multicolumn{2}{|l|}{ E0-5D German TTO } & \multicolumn{2}{|l|}{ тто } & \multicolumn{2}{|l|}{ VAS } \\
\hline & OR (95\% CI) & p Value & OR (95\% CI) & p Value & OR $(95 \% \mathrm{CI})$ & p Value & OR (95\% CI) & p Value \\
\hline Age & 1.27 (1.06 to 1.52$)$ & 0.010 & 1.13 (1.01 to 1.27$)$ & 0.038 & $1.06(0.94$ to 1.20$)$ & 0.350 & 0.98 (0.88 to 1.08$)$ & 0.659 \\
\hline Better eye treated & $2.59(0.21$ to 31.65$)$ & 0.456 & $1.97(0.31$ to 12.42$)$ & 0.468 & $0.06(<0.01$ to 1.05$)$ & 0.054 & 4.70 (0.83 to 26.47$)$ & 0.079 \\
\hline VA improvement (treated eye) & $3.89(0.46$ to 32.72$)$ & 0.212 & $3.88(0.69$ to 21.90$)$ & 0.124 & $0.08(0.01$ to 0.67$)$ & 0.020 & $0.43(0.07$ to 2.56$)$ & 0.355 \\
\hline VI status change & 7.92 (0.62 to 101.77$)$ & 0.112 & $1.05(0.16$ to 6.88$)$ & 0.957 & $9.08(0.79$ to 103.94$)$ & 0.076 & 0.72 (0.11 to 4.58$)$ & 0.731 \\
\hline
\end{tabular}

Emboldened values indicate statistical significance.

TTO, time trade-off; VA, visual acuity; VAS, visual analogue scale; VI, visual impairment.

Use of utility instruments comes with a range of inherent challenges. Whether utility measures are sensitive to changes in health status over a short period of time is debatable. ${ }^{12}$ Similarly, anchoring them for perfect vision-blindness rather than perfect health or death-is controversial ${ }^{9}$ as utility measures tend to produce lower utilities when anchored for blindness rather than death, which makes comparability across different illnesses and interventions difficult. ${ }^{9}$ Indeed, SG anchored for blindness was not associated with any of the assessed factors in our study. This, coupled with the high concordance of both measures, suggests that anchoring for death is appropriate as this yields universally comparable results. Another issue concerns obtaining utilities from an elderly sample with a short remaining life span and low willingness to trade any of it for an intervention. This is reflected in our study where most utility values were associated with age. The VAS, as a magnitude estimate of health, or in this case VRQoL, was highly correlated to the NEI-VFO-25 person measures, but not with other utility measures. Thus we would not recommend it to be used as the sole utility measure in any research scenario.

Better or worse eye treatment was not associated with improvements in utilities in our study. This may have implications for cost-effectiveness analyses which often assume a differential impact of better or worse eye treatment on utilities. Utility values used for economic evaluation in ophthalmology, in particular in the field of $\mathrm{AMD}$, are often deduced using approximations from clinical data (eg, visual acuity) rather than from directly collected utilities. ${ }^{13}$ Utilities directly derived from patients are very likely to differ from utility values inferred from visual acuity, as patients' preferences are vastly different even at the same level of visual acuity.

Vision functioning improved in our sample by four points, which is similar to the minimal clinically important difference for the non-Rasch adjusted NEI-VFO-25 (3-4 points in the composite score). ${ }^{14}$ The observed increase in visual functioning was similar regardless of whether the better or worse eye was treated, which corresponds to findings reported from the ANCHOR and MARINA studies. ${ }^{2}$

Strengths of our study include the use of a standard VRQoL instrument and a large array of utility tools to assess ranibizumab treatment effects in nvAMD, which has not been performed previously. Moreover, the use of three different population value sets to calculate the EQ-5D utilities is also important, especially given the varying findings in our study. Further strengths include the use of Rasch analysis to assess the psychometric properties of the German NEI-VFQ-25, and to produce interval-level measurements of visual functioning. Such a psychometric evaluation has not been done for the German version of the NEI-VFQ-25 to date. Our study setting in daily clinical routine better reflects actual treatment conditions and outcomes than highly standardised phase III clinical trials. The main limitation of our study is the small sample size, and uneven size of the subgroups for better and worse eye treatment, which may have diminished our ability to reveal significant associations and ensure that the associations we did find were not due to chance alone. Indeed, the wide CIs present for some of our data imply poor precision, and future trials are required to replicate the findings in a larger sample population. Another limitation is the short follow-up time; however, empirical evidence suggests that the largest treatment effect, if any, is to be expected at 3 months, and then either maintained or lost over the subsequent months. Indeed, other large randomised studies have shown similar increases in NEI-VFQ-25 visual functioning score at 3 months to that found in our study. $^{12}$

In conclusion, ranibizumab treatment for nvAMD leads to improvements in visual acuity, patient reported visual functioning and patient preferences, as elicited by TTO. Non vision-specific utility measures, or SG anchored for blindness, did not detect a change over time. Better or worse eye treatment was not associated with any of the patient reported utilities assessed, suggesting that gain in utility from better and worse eye treatment may be similar and that differential allocation of resources in cost-effectiveness analyses may not be warranted.

Acknowledgements We thank Steven Kymes and Walt Sumner (Washington University School of Medicine) for providing us with the X-Trade software for the estimation of standard gamble utilities.

Contributors Conception and design, or analysis and interpretation of data: $\mathrm{CWH}$, ASN, RPF, EF, AEH. Drafting the article or revising it critically for important intellectual content: RPF, EF, CWH, AK, MK, AW, AEH. Final approval of the version to be published: all authors.

Funding This work was supported by Novartis Pharma Germany (grant to $\mathrm{CH}$ ) and the German Research Council (DFG FI 1540/5-1, grant to RF). CERA receives Operational Infrastructure Support from the Victorian government.

Competing interests None.

Patient consent Obtained.

Ethics approval Ethics approval was provided by the Ethics Committee, University of Munich.

Provenance and peer review Not commissioned; externally peer reviewed.

\section{REFERENCES}

1. Chang TS, Bressler NM, Fine JT, et al. Improved vision-related function after ranibizumab treatment of neovascular age-related macular degeneration: results of a randomized clinical trial. Arch Ophthalmol 2007;125:1460-9.

2. Bressler NM, Chang TS, Suner IJ, et al. Vision-related function after ranibizumab treatment by better- or worse-seeing eye: clinical trial results from MARINA and ANCHOR. Ophthalmology 2010;117:747-56.e4.

3. Drummond MF, Sculpher MJ, Torrance GW, et al. Methods for the Economic Evaluation of Health Care Programmes. 3rd edn. Oxford: Oxford University Press, 2005.

4. Hirneiss C, Rombold F, Kampik A, et al. Visual quality of life after vitreoretinal surgery for epiretinal membranes. Ophthalmologe 2006;103:109-13. 
5. Mitchell P, Annemans L, White R, et al. Cost effectiveness of treatments for wet age-related macular degeneration. Pharmacoeconomics 2011;29:107-31.

6. Hirneiss C, Neubauer AS, Gass CA, et al. Visual quality of life after macular hole surgery: outcome and predictive factors. Br J Ophthalmol 2007:91:481-4.

7. Finger RP, Fenwick E, Marella $M$, et al. The impact of vision impairment on visionspecific quality of life in Germany. Invest Ophthalmol Vis Sci 2011:52:3613-19.

8. Hirneiss C, Rombold F, Kampik A, et al. Visual quality of life after vitreoretinal surgery for epiretinal membranes. Ophthalmologe 2006;103:109-13.

9. Lee BS, Kymes SM, Nease RF Jr, et al. The impact of anchor point on utilities for 5 common ophthalmic diseases. Ophthalmology 2008;115:898-903.e4.

10. Anon. EuroOol-a new facility for the measurement of health-related quality of life. The EuroOol Group. Health Policy 1990:16:199-208.
11. Marella M, Pesudovs K, Keeffe JE, et al. The psychometric validity of the NEI VFO25 for use in a low-vision population. Invest Ophthalmol Vis Sci 2010;51:2878-84.

12. Katz JN, Phillips CB, Fossel AH, et al. Stability and responsiveness of utility measures. Med Care 1994:32:183-8.

13. Bansback N, Czoski-Murray C, Carlton J, et al. Determinants of health related quality of life and health state utility in patients with age related macular degeneration: the association of contrast sensitivity and visual acuity. Qual Life Res 2007; 16:533-43

14. Submacular Surgery Trials Research Group. Evaluation of minimum clinically meaningful changes in scores on the National Eye Institute Visual Function Questionnaire (NEI-VFO) SST Report Number 19. Ophthalmic Epidemiol 2007;14:205-15 